$07 ; 11$

\title{
Сверхширокополосный генератор хаотических колебаний микроволнового диапазона с дополнительным нелинейным контуром
}

\author{
(c) Н.A. Максимов \\ Институт радиотехники и электроники им. В.А. Котельникова РАН (Фрязинский фрилиал), Фрязино, Московская обл., \\ Россия \\ E-mail: maksna49@mail.ru
}

Поступило в Редакцию 17 июня 2019г.

В окончательной редакции 20 августа 2019 г.

Принято к публикации 5 сентября 2019г.

Показана возможность генерации сверхширокополосных хаотических колебаний в диапазоне частот

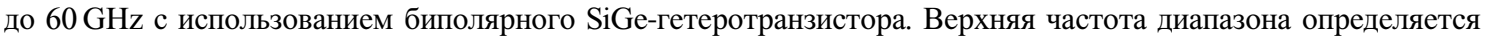
граничной частотой активного элемента генератора. Такая возможность появляется с введением в структуру генератора дополнительного нелинейного контура, в котором могут развиваться хаотические колебания вплоть до указанной частоты. Приводятся результаты экспериментального исследования одного из вариантов генератора на сосредоточенных элементах.

Ключевые слова: сверхширокополосные хаотические колебания, нелинейный контур, спектр, биполярный $\mathrm{SiGe-транзистор,} \mathrm{микроволновый} \mathrm{диапазон.}$

DOI: 10.21883/PJTF.2019.23.48721.17932

Развитие и рост количества радиоэлектронных устройств приводят к необходимости использования различных участков электромагнитного спектра с тенденцией продвижения в более высокочастотную его часть (вплоть до $100 \mathrm{GHz}$ ). Развитие коммуникационных технологий на основе сверхширокополосных хаотических сигналов, навигации, радиолокации и других задач прикладного характера требует создания источников таких сигналов с определенными требованиями для каждого из конкретных приложений [1-4]. В настоящей работе описывается подход к созданию источника хаотических колебаний с максимально возможным диапазоном генерации, характерным для гетеротранзистора BFP620F, который может быть применен и для более высокочастотных $p-n$-структур, в том числе и на базе кремнийгерманиевых ( $\mathrm{SiGe})$ технологий различного уровня [5].

В работе [6] описаны и экспериментально исследованы классический генератор Колпитца [7] и его модифицированный вариант упрощенной структуры с одним навесным реактивным элементом. В этих генераторах в качестве активного элемента использовался $\mathrm{SiGe}$ транзистор ВFР620F с граничной частотой $\sim 65 \mathrm{GHz}$. Показана возможность генерации в модифицированном варианте сверхширокополосных хаотических колебаний в СВЧ-диапазоне до $8 \mathrm{GHz}$ с шириной полосы генерации $\sim 7.4 \mathrm{GHz}$ по уровню $10 \mathrm{~dB}$, что значительно превышает диапазон генерации (с полосой $4.6 \mathrm{GHz}$ ) устройства, собранного по классической схеме Колпитца [7].

Спектр колебаний генераторов, рассмотренных в [6], можно значительно расширить, если добавить в схему пассивный нелинейный контур с использованием $p-n$-переходов транзистора ВFР620F и использовать его как выходной контур генератора.
Рассмотрим отдельно нелинейный колебательный контур. Известно [8], что при гармоническом воздействии на колебательный контур, содержащий нелинейную емкость $p-n$-перехода, в системе возбуждаются хаотические колебания. Такая динамика характерна для нелинейного контура как в области низких частот, так и в СВЧ-диапазоне [3,9]. В качестве нелинейного элемента можно использовать варакторный диод или один из $p-n$-переходов биполярного транзистора. В нашем случае использовался один из $p-n$-переходов (коллектор-база) транзистора ВFР620F, последовательно соединенный с индуктивностью $L=1 \mathrm{nH}$ [3]. При воздействии на такой контур внешним гармоническим сигналом достаточной амплитуды в нем происходит возбуждение сверхширокополосных хаотических колебаний. Так, при внешнем сигнале с частотой $8 \mathrm{GHz}$ и амплитудой $3 \mathrm{~V}$ в контуре развиваются хаотические колебания с довольно равномерным спектром от 2 до $20 \mathrm{GHz}$.

Резонансная частота контура $F_{r e s}=8 \mathrm{GHz}$ задается барьерной емкостью $p-n$-перехода при нулевом смещении на переходе и индуктивностью $L$, в данном случае равной $1 \mathrm{nH}$.

Развитие хаотических колебаний разной интенсивности в контуре возможно при различных частотах и амплитудах внешнего сигнала. Воздействие внешним гармоническим сигналом с частотой, близкой к резонансной частоте контура, и достаточной амплитудой вызывает развитие в контуре наиболее интенсивных широкополосных хаотических колебаний.

В основе подхода к созданию максимально сверхширокополосной автоколебательной системы с хаотическим поведением лежит замена однонаправленного воздействия на нелинейный контур сигнала 


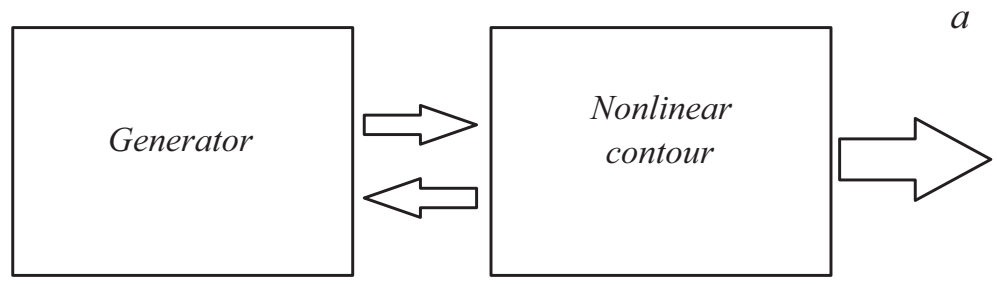

$b$

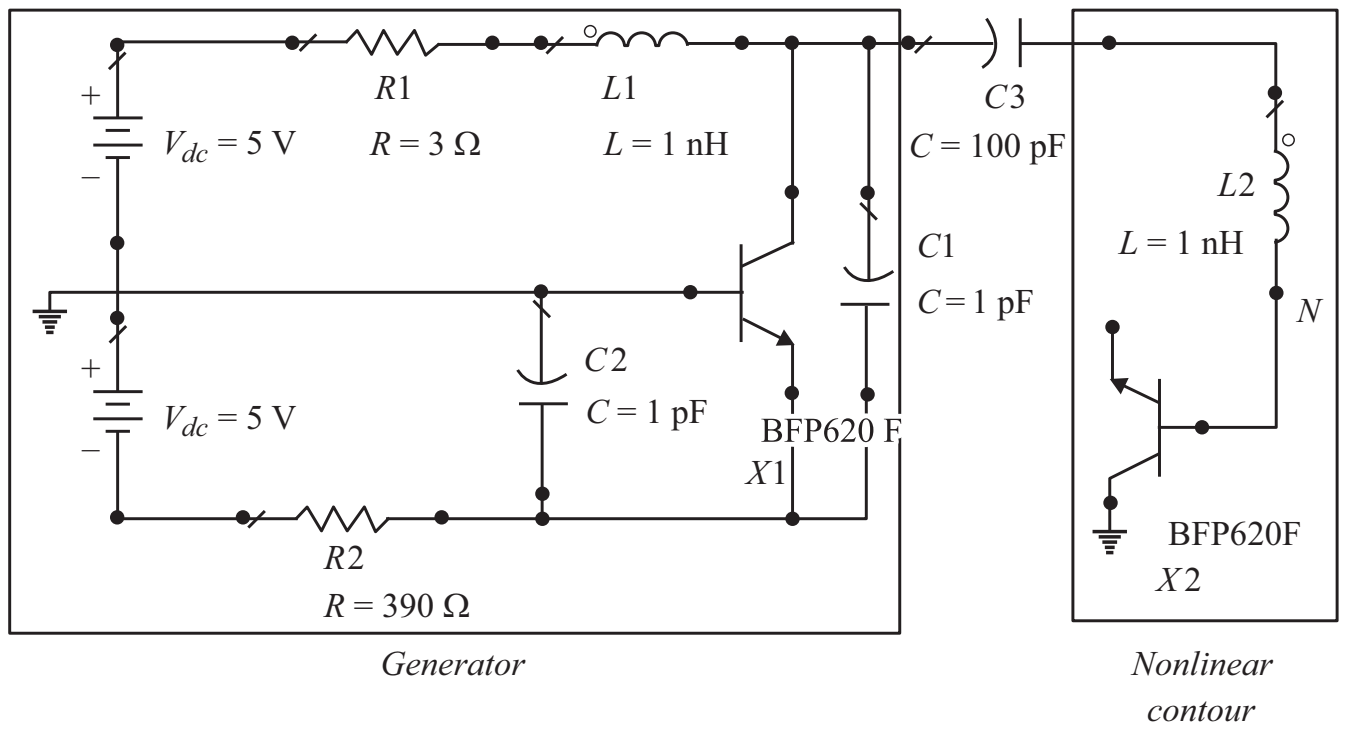

Рис. 1. $a$ - общая блок-схема генератора с дополнительным нелинейным контуром; $b$ - реальная схема генератора, структурно повторяющая блок-схему.

от внешнего источника самосогласованной системой генератор-нелинейный контур. Блок-схема предлагаемого генератора представлена на рис. $1, a$.

Общая блок-схема системы состоит из генератора и взаимно связанного с ним нелинейного контура. В такой системе происходит взаимное влияние этих структур друг на друга, что приводит к развитию хаотических колебаний с полосой генерации, определяемой добротностью возбужденного нелинейного контура. В качестве генератора может использоваться источник одночастотных или многочастотных колебаний, режим генерации которого меняется в результате обмена энергией с нелинейным контуром и может переходить в хаотический. Таким образом, благодаря взаимной связи первоначальное одночастотное или многочастотное воздействие на контур переходит в воздействие хаотическим сигналом, что в свою очередь приводит к исчезновению в спектре колебаний выделенных частот и его расширению. Выходной сигнал системы снимается с нелинейного контура. Достаточным условием развития хаоса в такой системе являются сильная взаимная связь между генератором и нелинейным контуром и воздействие сигналом генератора в окрестности резонансной частоты контура.

На рис. $1, b$ приведена реальная схема, построенная по принципу блок-схемы, в которой в качестве источника сигнала использовался генератор Колпитца на базе транзистора BFP620F, работающий в СВЧ-диапазоне. При указанных параметрах схемы на выходе этого генератора в автономном режиме наблюдался многочастотный сигнал. Нелинейный контур был построен с использованием коллекторного перехода транзистора ВFР620F и индуктивности $L 2=1 \mathrm{nH}$. Взаимная связь между блоком генератора и нелинейным контуром определялась величиной емкости $C 3=100 \mathrm{pF}$. При схемотехническом моделировании транзистор ВFР620F был представлен в виде модели Гуммеля-Пуна с учетом паразитных параметров.

Невысокая добротность нелинейного контура в режиме возбуждения и высокая граничная частота используемого $p-n$-перехода создают условия для существования сверхширокополосных колебаний в системе. Спектр хаотических колебаний на $p-n$-переходе нелинейного контура (в точке $N$ ) имеет верхнюю границу $\sim 60 \mathrm{GHz}$, которая является практически паспортной граничной частотой транзистора BFP620F (рис. 2,a).

Изменяя параметры генератора (в данном случае генератора Колпитца), можно влиять на характеристики выходного сигнала системы, а именно изменять неравномерность спектральной характеристики, управляя выходной мощностью по диапазону генерации. 

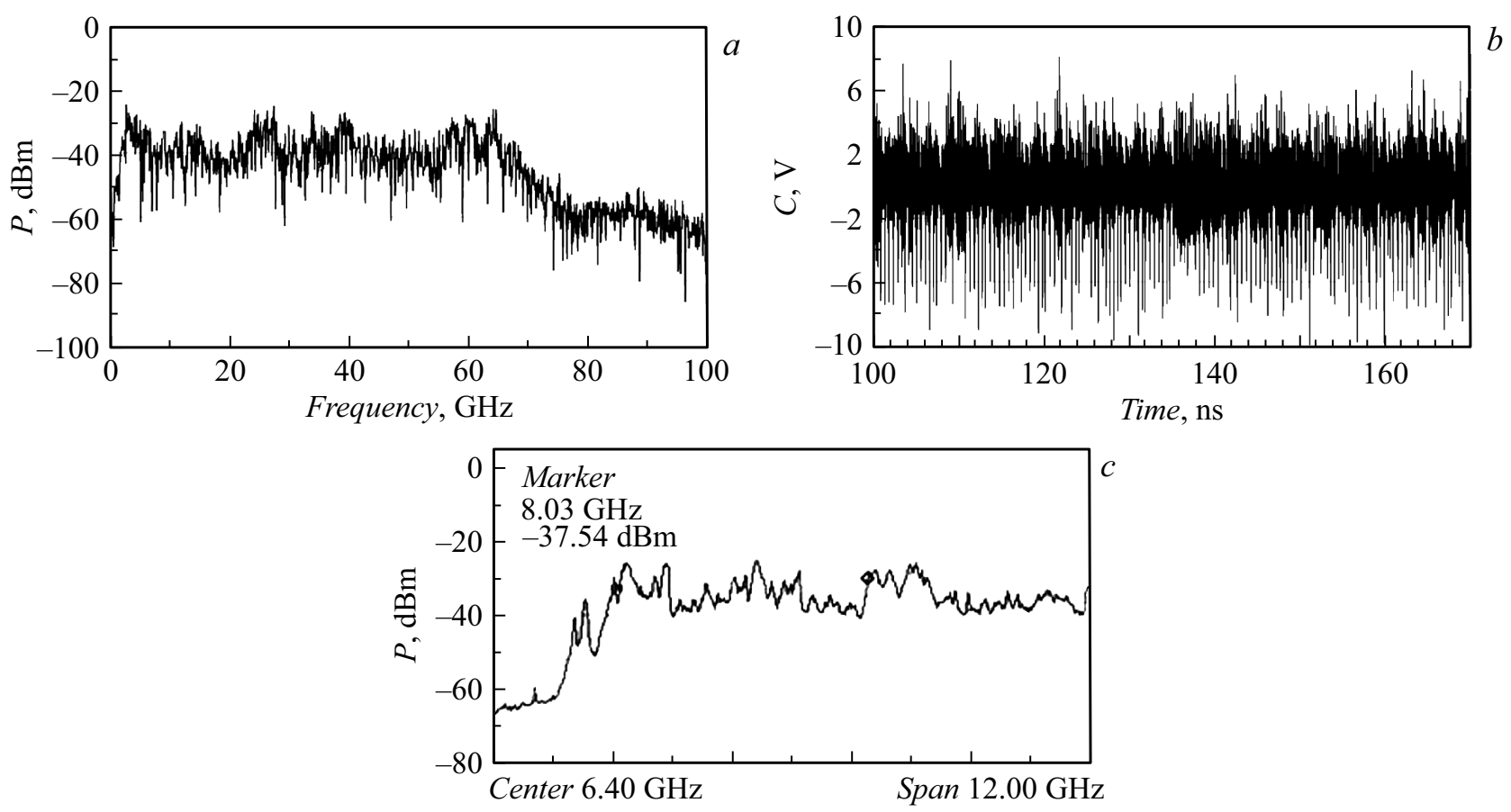

Рис. 2. $a-$ спектральная характеристика колебаний в точке $N$ нелинейного контура, схемотехническое моделирование; $b-$ реализация выборки колебаний во временно́м интервале $100-170 \mathrm{~ns} ; c-$ спектр выходного сигнала генератора, эксперимент.

При такой сверхширокополосности колебаний можно использовать как весь диапазон до $60 \mathrm{GHz}$, так и (с помощью фильтров) любой требуемый участок этого диапазона в зависимости от конкретной задачи. На рис. $2, b$ приведена реализация выборки во временно́й области $100-170 \mathrm{~ns}$, демонстрирующая случайный характер колебаний в контуре.

В соответствии с блок-схемой (рис. $1, a)$ был изготовлен и исследован экспериментальный макет генератора. В отличие от схемотехнической модели воздействующий генератор был собран по схеме Колпитца на базе транзистора BFP620F с одним источником питания. Параметры нелинейного контура такие же, как при моделировании. Топология создавалась на подложке материала RF-4 толщиной $0.5 \mathrm{~mm}$. Схема была собрана на ЧИП-элементах типоразмера 0402.

Динамика режимов генератора в зависимости от напряжения питания следующая: генерация возникает на частоте $\sim 8 \mathrm{GHz}$ при напряжении порядка $U=2 \mathrm{~V}$, далее при $U=2.4 \mathrm{~V}$ появляются субгармоники и гармоники основной частоты. Этот режим сменяется многочастотным с зонами хаоса у основания спектральных составляющих при $U=2.6 \mathrm{~V}$, а при $U=2.8 \mathrm{~V}$ и выше система переходит в режим хаотических колебаний (рис. 2,c). В физическом эксперименте было установлено, что в генераторе возможно существование сверхширокополосных хаотических режимов в диапазоне частот от $2 \mathrm{GHz}$ до более $12 \mathrm{GHz}$ с энергетической эффективностью $\sim 5 \%$. Диапазон генерации хаотических колебаний, полученный в эксперименте, значительно превышает диапазон таких колебаний, описанный в ра- боте [6]. Более низкая, чем при моделировании, верхняя частота диапазона генерации связана с технологией изготовления устройства.

На основании проведенного исследования схемотехнической модели и экспериментального макета можно сделать заключение, что добавление нелинейного пассивного элемента в схему генератора в виде колебательного контура с $p-n$-переходом позволяет значительно расширить диапазон генерации хаотических колебаний вплоть до граничной частоты активного элемента, содержащего этот $p-n$-переход. Для дальнейшего развития предлагаемого подхода необходимо при создании устройства использовать новые кремний-германиевые интегральные технологии.

\section{Финансирование работы}

Исследование проведено при выполнении планового госзадания.

\section{Конфликт интересов}

Автор заявляет, что у него нет конфликта интересов.

\section{Список литературы}

[1] Дмитриев А.С., Панас А.И. Динамический хаос. Новый носитель информации для систем связи. М.: Физматлит, 2002. $252 \mathrm{c}$.

[2] Chong C., Young S.K. // IEEE Trans. Vehicular Technol. 2008. V. 57. N 3. P. 67-70. 
[3] Дмитриев А.С., Ефремова Е.В., Максимов Н.А., Панас А.И. Генерация хаоса. М.: Техносфера, 2012. $424 \mathrm{c}$.

[4] Li J.X., Wang Y.C., Ma F.C. // Nonlinear Dyn. 2013. V. 72. N 3. P. 575-580.

[5] Ефремова Е.В. // Письма в ЖТФ. 2018. Т. 44. В. 9. С. 26-33.

[6] Максимов Н.А., Панас А.И. // Письма в ЖТФ. 2017. Т. 43. B. 3. C. $88-94$.

[7] Kennedy M.P. // IEEE Trans. Circ. Syst. 1: Theory and Applications. 1994. V. 41. N 11. P. 771-774.

[8] Linsay P.S. // Phys. Rev. Lett. 1981. V. 47. N 19. P. 1349-1352.

[9] Максимов Н.А., Кислов В.Я. // Радиотехника и электроника. 1997. Т. 42. № 12. С. 1487-1492. 